\title{
BMJ Open Hearing Norton Sound: a community randomised trial protocol to address childhood hearing loss in rural Alaska
}

\author{
Susan D Emmett, ${ }^{1,2}$ Samantha Kleindienst Robler, ${ }^{3}$ Nae-Yuh Wang, ${ }^{4,5}$ \\ Alain Labrique, ${ }^{6}$ Joseph J Gallo, ${ }^{7}$ Philip Hofstetter ${ }^{8}$
}

To cite: Emmett SD, Robler SK, Wang N-Y, et al. Hearing Norton Sound: a community randomised trial protocol to address childhood hearing loss in rural Alaska. BMJ Open 2019:9:e023078. doi:10.1136/ bmjopen-2018-023078

- Prepublication history for this paper is available online. To view these files, please visit the journal online (http://dx.doi org/10.1136/bmjopen-2018023078).

Received 22 March 2018 Revised 26 July 2018

Accepted 28 September 2018

Check for updates

(C) Author(s) (or their employer(s)) 2019. Re-use permitted under CC BY-NC. No commercial re-use. See rights and permissions. Published by BMJ.

For numbered affiliations see end of article.

Correspondence to

Dr Susan D Emmett susan.emmett@duke.edu

\section{ABSTRACT}

Introduction The population in rural Alaska experiences a disproprionately high burden of infection-mediated hearing loss. While the state mandates school hearing screening, many children with hearing loss are not identified or are lost to follow-up before ever receiving treatment. A robust, tribally owned healthcare system exists in Alaska, but children with hearing loss must first be identified and referred for existing infrastructure to be used. This trial will evaluate a new school hearing screening and referral process in rural Alaska, with the goal of improving timely identification and treatment of childhood hearing loss. Methods and analysis Comparative effectiveness community randomised trial testing digital innovations to improve school hearing screening and referral in 15 communities in the Norton Sound region of northwest Alaska, with data collection from October 2017 to February 2020. All children (K-12) attending school in Bering Strait School District with parental informed consent and child assent will be eligible (target recruitment $n=1500$ ). Participating children will undergo both the current school hearing screen and new mobile health (mHealth) screen, with screening test validity evaluated against an audiometric assessment. Communities will be cluster randomised to continue the current primary care referral process or receive telemedicine referral for follow-up diagnosis and treatment. The primary outcome will be time to International Statistical Classification of Diseases, 10th Revision, ear/hearing diagnosis from screening date, measured in days. Secondary outcomes will include: sensitivity and specificity of current school and mHealth screening protocols measured against a benchmark audiometric assessment (air and bone conduction audiometry, tympanometry and digital otoscopy); hearing Ioss prevalence; hearing-related quality of life; and school performance (AIMSweb). Intention-to-treat analysis will be used.

Ethics and dissemination This study has been approved by the Institutional Review Boards of Alaska Area, Norton Sound and Duke University and is registered on clinicaltrials.gov. Results will be distributed with equal emphasis on scientific and community dissemination. Trial registration number NCT03309553; Pre-results.

\section{INTRODUCTION}

Hearing loss impairs childhood development, restricts educational achievement

\section{Strengths and limitations of this study}

- A strength of this study is that the intervention was developed with Alaska stakeholder and community input, including parents, teachers, administrators, community health aides, children and community leaders, to ensure that it represents a culturally relevant solution to address undiagnosed childhood hearing loss in rural Alaska.

- This study will provide the first empirical evidence on the effectiveness of current school screening programmes in the state of Alaska and represents a unique opportunity to evaluate a new school screening protocol that uses mobile health (mHealth)based screening tools and telemedicine referral in a remote region of Alaska where telemedicine has already been standard practice for triage of health concerns for over 15 years.

- This study will provide much-needed, current epidemiological data on childhood hearing loss in Alaska.

- Screening and referral interventions are by nature meant to be applied to communities as a whole, so the cluster randomised design is a logical choice. However, a cluster randomised trial has lower statistical power and precision than an individually randomised trial with the same number of individual participants and has to address typical challenges such as selection bias, imbalance between study arms and generalisability accompaning a cluster randomised design. The time frame of annual screening and referral interventions over two academic years limits the possibility of adapting other design features, such as step-wedge or waitlist control for the telemedicine referral process.

While aspects of this intervention are designed specifically for the Alaskan context, the combination of mHealth screening and telemedicine referral could be replicated to address childhood hearing loss disparities in other remote regions of the world.

and compromises future earning potential. ${ }^{1-9}$ Rural Alaska, where the population is primarily Alaska Native, experiences a disproportionately high burden of infection-mediated hearing loss compared with the general US population. ${ }^{10-14}$ 
Barriers to care exacerbate the alarmingly high prevalence of hearing loss in rural Alaska. Spanning 586000 square miles, $75 \%$ of Alaskan communities are not connected to a hospital by road, necessitating travel by plane or boat to be seen by a specialist. ${ }^{15}$ Population sparsity (1.1 per square mile) and a low ratio of doctors to residents contribute to pervasive delays in care ${ }^{1516}$ In response to these challenges, tribally owned regional healthcare systems have developed an extensive network of village health clinics that are staffed by community health aides (CHAs) to provide basic healthcare services in remote communities. ${ }^{17} 18$ Innovative telemedicine technology has been adopted in Alaska over the past 15 years to improve access to care. A statewide telemedicine network allows consults initiated in communities to be read remotely by specialists in an asynchronous fashion. ${ }^{15}$ 19-22 Through telemedicine, a specialist can direct care provided by CHAs in the community or expedite travel to a regional centre if an in-person visit, imaging or surgery is required, thus bypassing delays in primary care. Early in its adoption, asynchronous or 'store and forward' telemedicine consultation was validated as equivalent to in-person examination for ear and hearing problems. ${ }^{23-25}$

The state of Alaska mandates school hearing screening, an important step in identifying children with hearing loss. ${ }^{26}$ It is unclear, however, how effective the current screening protocols are in identifying children with hearing loss in a timely manner, and loss to follow-up in the referral stage is a persistent problem. While telemedicine technology has been used to triage ear and hearing problems in communities for many years, it has never been used in the school hearing screening referral process. This trial builds on the existing school screening mandate and telemedicine network in Alaska to compare two school screening and referral processes in the Bering Strait School District (BSSD) of the Norton Sound region of northwest Alaska. If proven effective, the new mobile health (mHealth) screening and expedited telemedicine referral process evaluated in this study could be implemented in school districts across the state of Alaska. Results could be generalisable to populations living in other remote locations in the USA and worldwide.

\section{Gaps in the evidence}

Multiple gaps exist in quantifying prevalence, risk factors and consequences of childhood hearing loss in rural Alaska, as well as effectiveness of screening and referral protocols in identification and management of childhood hearing loss in remote environments. ${ }^{11} 1214212728$ The little epidemiological data available suggest otitis media prevalence of $30 \%-78 \%$ in Alaska Native children, with hearing loss in $31 \% .^{1027} 28$ This is in stark contrast to the $\sim 1.7 \%-5 \%$ prevalence of childhood hearing loss in the general US population, with studies in both populations defining hearing loss as pure-tone average (PTA) $>25 \mathrm{~dB}(500 \mathrm{~Hz}, 1000 \mathrm{~Hz}$ and $2000 \mathrm{~Hz}) .{ }^{1029}$ With much of the Alaska data now over 50 years old, there is an urgent need for updated epidemiological data. Risk factors that contribute to high hearing loss prevalence in rural Alaska need to be further elucidated. Low socioeconomic status (SES) has been associated with hearing loss previously, ${ }^{8912}$ but measures of SES used in studies in the continental USA do not account for the richness of culture and health associated with Alaska Native traditions of subsistence and lifestyle (ie, living off the land and sea). Data are needed on the impact of environmental factors on hearing loss, from lack of running water in some rural Alaska communities to household crowding and indoor air pollution. ${ }^{1130-32}$

An important method of identifying and managing otitis media and subsequent hearing loss in children is school hearing screening. ${ }^{33}$ However, current screening programmes in Alaska have never been studied to assess sensitivity and specificity. Loss to follow-up from referrals is anecdotally a major problem in the school screening process, yet tracking the proportion of children referred from screening who actually go on to be diagnosed and treated has never been done.

\section{Objective}

The objective of the Hearing Norton Sound study is to evaluate a new school hearing screening and referral process in rural Alaska, with the goal of improving timely identification and treatment of childhood hearing loss. The study is funded by the Patient-Centered Outcomes Research Institute, a non-governmental organisation that supports patient-centred comparative effectiveness research and represents a collaboration between Norton Sound Health Corporation, Duke University and Johns Hopkins University. Stakeholder and community involvement are integral to this mixed methods community randomised trial and are described in depth in the companion protocol. ${ }^{34}$ This protocol focuses specifically on the community randomised trial, with the specific aim of:

\section{Aim}

Complete a comparative effectiveness community randomised trial of two school-based screening and referral processes in rural Alaska that evaluates time to diagnosis (primary outcome), sensitivity and specificity of screening against audiometric evaluation, prevalence of hearing loss by audiometric evaluation, hearing-related quality of life (HEAR-QL) and school performance (secondary outcomes).

\section{Hypothesis 1}

Expedited telemedicine referral will reduce time to diagnosis compared with the current primary care referral process, leading to more timely treatment of childhood hearing loss.

\section{Hypothesis 2}

mHealth school screening will be more sensitive than the current school screening protocol. 
Exploratory sequential

\section{METHODS AND ANALYSIS \\ Overview of study design}

The Hearing Norton Sound study is a mixed methods comparative effectiveness community randomised trial on school-based hearing screening and referral process (figure 1). Qualitative components of the study, including community engagement in the form of focus groups and semi-structured interviews to gain community and stakeholder perspectives on the intervention, are described in the companion protocol. ${ }^{34}$ The study design was selected with two primary intentions: (1) to maintain a real-world environment that will allow study results to be scaled up across the state and (2) to be highly sensitive to cultural concerns regarding research in Alaska Native communities. The framework for the community randomised trial is summarised in table 1 .

\section{Patient and public involvement}

Patient and community involvement is central to the design, execution and dissemination of the Hearing Norton Sound study and is described in depth in the companion protocol. ${ }^{34}$

\section{Study population, setting and eligibility}

The BSSD, comprised of 15 communities in the Norton Sound region, has been selected for the study. The study will be conducted in the school and clinic associated with each of these 15 communities. All school-aged children, from kindergarten through 12th grade, will be eligible to participate. Compared with the general US population, prevalence of hearing loss for Alaska Native children is high across this entire age range, so inclusion of all grades is important.

\section{Recruitment}

Parents of all children enrolled in BSSD will receive information about the study and a written informed consent during the school intake process. This is standard practice for health interventions provided in the school setting in Alaska. A sociodemographic survey tailored to reflect the rural Alaska environment will also be included for parental completion. Study enrolment will occur over two academic years and will happen in each school on hearing screening day. All children with signed parental consent who are present on the day of screening will be enrolled. Verbal assent will be obtained from each child in a one-on-one environment prior to participation. Verbal and non-verbal cues will be used to determine a child's level of willingness to participate (eg, eyebrow raise indicates yes in this population). Despite parental consent, should a child indicate that they do not want to participate, they will not be enrolled. Children will be allowed to stop study involvement at any time.

We anticipate reaching $85 \%$ of target enrolment $(n=1500)$ within the first 12 months of the trial. The intention of the study is to maintain a real-world environment where all children from kindergarten to 12th grade undergo school screening. Therefore, enrolment over two academic years is necessary to be inclusive of transferring students and new kindergartners matriculating in year 2 of the trial. 
Table 1 PICOTS framework for the comparative effectiveness community randomised trial

Population Children attending kindergarten through 12th grade (4-21 years of age) in the Bering Strait School District (BSSD) in the Norton Sound region of Alaska. Up to 1900 students in 15 communities will be eligible to participate.

Intervention Mobile health (mHealth) hearing screening and expedited telemedicine referral.

Comparators There will be two comparators for screening and referral in this trial. Each child in participating communities will undergo the current school screening protocol and new mHealth screening, with screening test validity evaluated against clinical audiometric evaluation. Communities will be cluster randomised to: (1) primary care referral or (2) expedited telemedicine referral. Both referral pathways have been used for many years for management of ear disease in Alaska; however, telemedicine has not previously been integrated into the school hearing screening referral process.

Outcomes

Timing

The primary outcome will be time to International Statistical Classification of Diseases, 10th Revision, ear/hearing diagnosis from the date of screening, measured in days. Secondary outcomes will include sensitivity and specificity of screening protocols against a benchmark audiometric evaluation, prevalence of hearing loss (pure-tone average $>25 \mathrm{~dB}$ at $0.5 \mathrm{kHz}, 1 \mathrm{kHz}, 2 \mathrm{kHz}$ and $4 \mathrm{kHz}$ ) by audiometric evaluation, hearing-related quality of life (HEAR-QL) and school performance (AIMSweb).

School screenings will be performed annually for 2 years. The current school screen, mHealth screen, audiometric evaluation and HEAR-QL administration will occur on school-screening day. AIMSweb testing occurs in all BSSD schools three times annually. Follow-up, in the form of chart review of the multiorganisational electronic medical record used by Norton Sound, will continue for 9 months after each annual screening.

Setting Schools and clinics in remote Alaska communities in the Norton Sound region.

PICOTS, population, intervention, comparators, outcomes, timing, setting.

\section{Randomisation and masking}

All enrolled school-aged children in each participating community will undergo the current school screening protocol, the new mHealth screening protocol and audiometric evaluation on hearing screening day. A cluster-randomised design will be used to randomise communities to receive primary care referral or expedited telemedicine referral, such that all children from a single community will undergo the same referral process. Randomised referral assignments will be computer generated by the study statistician, stratified by four strata based on the combination of school sizes and geographic location. All possible permutations of assignment results under a stratified randomisation with block size of 2 will be generated. Selection probabilities for these possible assignments will be inversely weighted so that assignments creating greater differences of school sizes between arms (within stratum and overall) are downweighted and less likely to be generated during randomisation. The set of randomised assignments will then be randomly generated according to this weighted probability distribution.

Communities will each be assigned an identification (ID) code within their geographic strata by the leadership team in Alaska. The study statistician at Johns Hopkins will generate randomised assignments based on the community ID without the knowledge of community identities. At the day of randomisation, after all communities become eligible for the trial (ie, $50 \%$ of parental inform consents per community have been obtained), the secure-stored ID list and the independently securestored randomisation list will be unsealed and matched according to community ID codes to carry out the randomisation assignment.
The randomisation assignments will be kept confidential within the study team until hearing screening day, when masking the referral process assignment will no longer be possible. All study team members will be trained and reminded of the importance of randomisation concealment so that families' willingness to participate in the trial will not be influenced by the knowledge of the referral assignment before enrolling in the study. In addition, all outcome assessors, including audiologists and ear, nose and throat (ENT) surgeons reading telemedicine consults within the Alaska Native healthcare system and study team members performing medical record abstraction, will be masked to intervention allocation. Study team members who read telemedicine consults as a part of their clinical responsibilities will abstain from reading any study-related consults. The results of the school screen, mHealth screen and audiometric assessments will also be masked, such that study team members performing the mHealth screen or audiometric assessment will be masked to the other results.

\section{Hearing screening protocols and referral intervention}

Our stakeholders and community members feel strongly that all enrolled children should derive benefit from enhanced school screening in the study and that all children in a community should undergo the same referral process. We have therefore carefully crafted a study design to honour these wishes. All enrolled children will undergo the current school screening protocol and the mHealth screening protocol, as well as a benchmark audiometric assessment, on school screening day. The referral process will be cluster randomised by community, such that all enrolled children in a community will either receive the 
current primary care referral process or the expedited telemedicine referral intervention. Here we describe the screening and audiometric protocols, followed by the randomised referral pathways.

\section{Hearing screening protocols}

\section{Current screening protocol}

In BSSD, students in grades K-5, 7, 9 and 11, along with children in any grade who referred screening the previous year, are screened annually using the Natus/Bio-Logic AuDX distortion product otoacoustic emission hearing screening. Screening is automated and involves school staff placing a soft tip in the ear and recording a pass or refer for each ear. Emissions are measured at $2 \mathrm{kHz}, 3$ $\mathrm{kHz}, 4 \mathrm{kHz}$ and $5 \mathrm{kHz}$ in each ear using an overall pass/ refer criteria in which three of four frequencies must meet predetermined response conditions, as published by Gorga and colleagues. ${ }^{35}$ Rescreening does not occur in this automated protocol. Failure to meet overall pass criteria in one or both ears will generate a referral, with referral pathways described in the following section. Screening is typically performed by special education teachers and staff in various classroom environments. The current screening does not specifically assess middle ear function, an essential component of an ear health evaluation in an environment where otitis media is highly prevalent. For the purpose of the trial, all students from all grades will be screened by teachers using the AuDX device annually for 2 years. This will allow direct comparisons of sensitivity and specificity of the screening protocols in all age groups.

\section{mHealth screening protocol}

The mHealth school screening protocol was selected based on systematic reviews ${ }^{36}$ and practice guidelines ${ }^{33}$ for school screening, taking into account the considerable role of chronic otitis media in childhood hearing loss in rural Alaska. mHealth screening will be coordinated with regularly planned school screenings so that the two screens occur on the same day, eliminating seasonal and temporal variation in screening results. The mHealth screening protocol will include pure-tone screening at standard frequencies using hearScreen, a validated smartphone-based hearing screen (hearX Group, South Africa), and an assessment of middle ear function through tympanometry (Otometrics, Denmark). New Zealand, with a similarly high prevalence of otitis media and hearing loss in Maori and Pacific Island populations, has incorporated tympanometry into their national screening protocol. ${ }^{37}$ Members of the study team who are not trained audiologists will perform the mHealth screen for all participating children. The screening protocol that will be employed has been validated with traditional audiometric equipment as $100 \%$ sensitive and $97 \%$ specific during a screening of 3510 children in British Columbia ${ }^{38}$ :

Pure-tone screening at $1 \mathrm{kHz}, 2 \mathrm{kHz}$ and $4 \mathrm{kHz}$ at $20 \mathrm{~dB}$ with a validated mHealth smartphone screen. ${ }^{39-42} \mathrm{~A}$ tone will be presented at each frequency to each ear.
If a child does not respond to a tone, a rescreen at that frequency will be performed at the end of the screening. Failure to respond to a tone at any frequency in either ear will generate a referral. ${ }^{33}$

Tympanometry screening. Type B (flat) tympanogram or negative pressure $<-200 \mathrm{~mm}$ decapascal (daPa) will generate a referral. ${ }^{38}$

\section{Audiometric assessment}

During screening day, each participating child will also undergo an audiometric assessment to determine the sensitivity and specificity of the current school screening and mHealth screening protocols. This assessment will be performed using Shoebox, a validated mHealth tablet audiometer (Shoebox Audiometry, Clearwater Clinical, Canada), Otometrics tympanometer (Denmark) and Otometrics Otocam digital otoscope (Denmark). Study team members who are trained audiologists will perform this assessment. They will be blinded to the results of the mHealth and school screens. Otoscopy is important for diagnosing ear conditions requiring treatment in a population with a high prevalence of otitis media, but it requires clinical skills and training that are not practical for implementation in a large-scale screening programme. We have therefore included otoscopy within our audiometric assessment as a component of the benchmark evaluation instead of incorporating it into the mHealth screening itself.

Air and bone conduction pure-tone audiometry using a validated mHealth tablet audiometer at $0.5 \mathrm{kHz}$, $1 \mathrm{kHz}, 2 \mathrm{kHz}$ and $4 \mathrm{kHz}^{43}$ A referral will be generated for PTA $>25 \mathrm{~dB}$ or a threshold $>30 \mathrm{~dB}$ in a single frequency. Bone conduction will be performed at $0.5 \mathrm{kHz}, 1 \mathrm{kHz}, 2 \mathrm{kHz}$ and $4 \mathrm{kHz}$ if hearing loss reaches the threshold for referral. Prevalence of hearing loss (PTA $>25 \mathrm{~dB}$ at $0.5 \mathrm{kHz}, 1 \mathrm{kHz}, 2 \mathrm{kHz}$ and $4 \mathrm{kHz}$ ) will be calculated from this audiogram.

Diagnostic tympanometry. Type B (flat) tympanogram or negative pressure $<-200 \mathrm{daPa}$ will generate a referral.

Digital otoscopy. Digital images of bilateral tympanic membranes will be taken. Pathological findings will generate a referral at the discretion of the audiologist.

Schools will generate a list of all children referred by the current screening protocol, as is already standard practice in each school. The study team will generate a list from the mHealth screen of all enrolled children who refer either by pure-tone screening or tympanometry. If a child passes the current and mHealth screening protocols but is noted during the audiometric assessment to have conditions requiring treatment, they will also be flagged for referral. Referral lists will be reconciled at the conclusion of the screening day to ensure that all families of children who refer will receive a single notification. Once a reconciled referral list has been developed by the study team, it will be handed over to local school leadership and clinic staff to initiate and manage the referral 

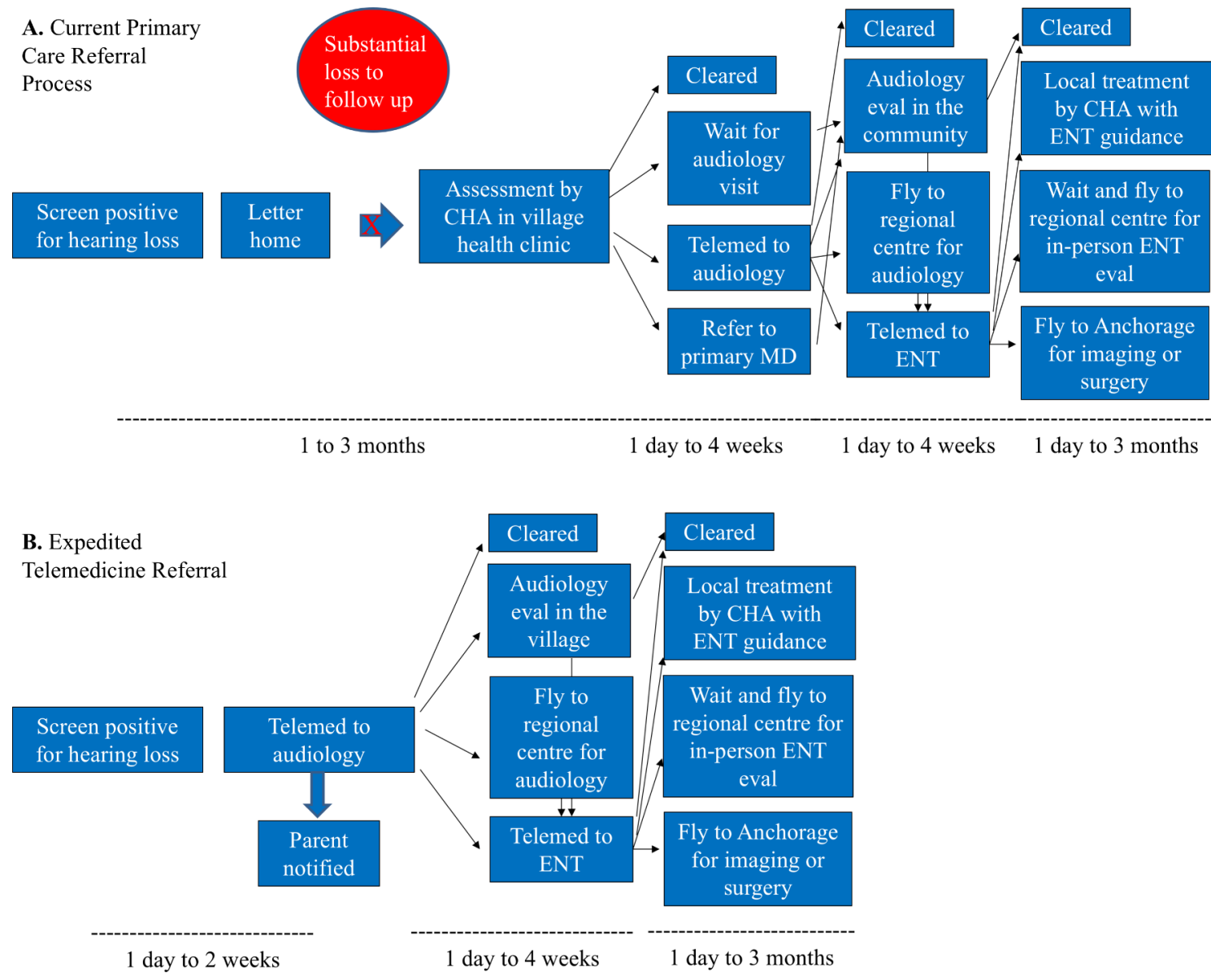

Figure 2 Primary care $(A)$ and expedited telemedicine $(B)$ referral pathways for diagnosis and treatment of childhood hearing loss in rural Alaska. CHA, community health aide; ENT, Ear, nose and throat surgery.

process. This hand-off is done to maintain a real-world environment that enhances generalisability of the study for future implementation in Norton Sound and other regions of Alaska.

\section{Intervention arms}

Communities will be randomised to the current primary care referral process or expedited telemedicine referral. Figure 2 highlights the complexity of the diagnosis and treatment pathway for childhood hearing loss in rural Alaska communities, with multiple branch points, wait times and potential need to travel for higher levels of care. The key differences between current (figure 2A) and expedited telemedicine referral processes (figure 2B) can be summarised by two fundamental issues we have identified with the current process: (1) children who refer screening can be lost to follow-up, preventing them from ever entering the healthcare system for diagnosis and treatment, and (2) despite the integration of telemedicine in village health clinics for many years, telemedicine has never been systematically applied to expedite school hearing screening referral.

\section{Current primary care referral process}

In communities randomised to the current primary care process, families will be notified if their children screen positive in exactly the same method each school had been using previously. This process is managed by the school and involves a letter home to the parents, either sent with the child or by mail, requesting that the parent/caregiver bring the child to the village health clinic for evaluation by a CHA. This assessment can lead to multiple treatment pathways, including waiting for audiology field clinic, telemedicine consultation to audiology or referral to a primary care physician. ENT surgery becomes involved in the treatment pathway if recommended by audiology (figure 2A). In addition to letters home requesting that parents bring children into clinic for evaluation, a list of referred children is also given to the Department of Audiology at Norton Sound Health Corporation. Audiology staff reach out to families to schedule appointments during the next available audiology clinic.

\section{Expedited telemedicine referral}

In communities randomised to the expedited telemedicine intervention, parents of children who screen positive will receive a phone call from the school or the clinic notifying them of the day and time of their child's telemedicine consultation appointment. Appointments will be made with CHAs who have dedicated time blocked off to perform referral telemedicine consults to audiology 
(figure 2B). Participating children who refer will be transported to clinic for their appointment with adult chaperones. Parents will be encouraged but not required to attend, except for children grades 2 and younger, for whom parental participation will be required. Thus, the expedited telemedicine referral pathway will systematically apply telemedicine consultation to audiology for all referrals and does not require the presence of a parent for completion. Similar to the current primary care referral process, ENT surgery will become involved in the treatment pathway if recommended by audiology.

Schools will manage the expedited telemedicine referral process jointly with clinic staff. The role of the study team is simply to provide a list to local school leadership and clinic staff of students being referred.

Non-participating children in communities assigned to the expedited telemedicine intervention arm will receive standard referral following the current primary care referral process. All services provided at the clinic for the purposes of clinical care for Alaska Natives, including the telemedicine intervention, will be covered by Indian Health Service.

It is important to note that all communities participating in this study have telemedicine within village health clinics that is routinely used by CHAs for triage and management of health problems, including ear-related complaints. While telemedicine is not routinely used for school hearing screening referrals at the current time, it will remain available to CHAs to use at their discretion throughout the course of the study, maintaining a realworld environment where some crossover between the study arms may occur. We will monitor the use of telemedicine through chart reviews in the multiorganisational electronic medical record (EMR) used by Norton Sound Health Corporation that will be conducted on all participating children who refer. Chart reviews will be completed within 9 months of the date of school hearing screening. If we find a child has been lost to follow-up through chart review, we will contact the families at that time and expedite diagnosis and treatment as needed.

\section{Outcome measures}

Primary outcome: time to diagnosis

Randomised trial outcomes are summarised in table 2. Time to International Statistical Classification of Diseases, 10th Revision (ICD-10), ear/hearing diagnosis from the date of screening, measured in days, will be used to assess time to entry into the healthcare system for the two referral pathways. All ear/hearing diagnoses in the Norton Sound EMR will be included, from a CHA examination or primary care provider to audiologist or ENT physician consultation, with time to entry defined by the first ear/hearing diagnosis recorded in the EMR after the date of screening. The inclusion of all types of providers reflects the current Alaska healthcare system, which exists in a continuum from village health clinics to regional centres and the tertiary referral hospital. The multiorganisational EMR used by Norton Sound is also used by the tertiary referral hospital in Anchorage where surgical ENT care is provided, enabling complete review of the entire diagnosis and treatment pathway. Based on preliminary data, we hypothesise that time to diagnosis will decrease in the expedited telemedicine referral communities compared with primary care referral communities (hypothesis 1). ${ }^{161922}$

\section{Secondary outcomes}

Sensitivity and specificity of the screening protocols will be measured against a benchmark audiometric assessment, including air and bone conduction audiogram, tympanometry and digital otoscopy. ${ }^{44-47}$ The accepted definition of hearing loss from the WHO will be used $(\mathrm{PTA}>25 \mathrm{~dB}$ over $0.5 \mathrm{kHz}, 1 \mathrm{kHz}, 2 \mathrm{kHz}$ and $4 \mathrm{kHz}){ }^{48}$ We have chosen to use the standard WHO definition of hearing loss instead of disabling childhood hearing loss (PTA $>30 \mathrm{~dB}$ over $0.5 \mathrm{kHz}, 1 \mathrm{kHz}, 2 \mathrm{kHz}$ and $4 \mathrm{kHz}$ ) because of ample evidence that mild hearing loss has a

Table 2 Quantitative outcomes for the community randomised trial

\begin{tabular}{|c|c|c|c|}
\hline Outcome & Definition & $\begin{array}{l}\text { Method for } \\
\text { measurement }\end{array}$ & Time measured \\
\hline Time to diagnosis (primary) & $\begin{array}{l}\text { Days from date of screening to ear/ } \\
\text { hearing ICD-10 diagnosis in EMR. }\end{array}$ & Chart review. & $\begin{array}{l}\text { Up to } 9 \text { months from date of } \\
\text { screening. }\end{array}$ \\
\hline $\begin{array}{l}\text { Sensitivity and specificity of } \\
\text { screening }\end{array}$ & $\begin{array}{l}\text { Sensitivity and specificity of school } \\
\text { and mHealth screening protocols } \\
\text { compared with benchmark audiometric } \\
\text { evaluation. }\end{array}$ & $\begin{array}{l}\text { School screen, mHealth } \\
\text { screen and audiometric } \\
\text { evaluation. }\end{array}$ & Screening day. \\
\hline Hearing loss prevalence & $\begin{array}{l}\text { Pure-tone average }>25 \mathrm{~dB}(0.5 \mathrm{kHz} \text {, } \\
1 \mathrm{kHz}, 2 \mathrm{kHz} \text { and } 4 \mathrm{kHz}) \text {. }\end{array}$ & Audiometric evaluation. & Screening day. \\
\hline $\begin{array}{l}\text { Hearing-related quality of } \\
\text { life (HEAR-QL) }\end{array}$ & Validated HEAR-QL child self-report. & HEAR-QL. & Screening day. \\
\hline School performance & $\begin{array}{l}\text { Performance on validated, districtwide } \\
\text { AIMSweb testing. }\end{array}$ & AIMSweb. & $\begin{array}{l}\text { Administered by schools } \\
\text { three times annually. }\end{array}$ \\
\hline
\end{tabular}

EMR, electronic medical record; ICD-10, International Statistical Classification of Diseases, 10th Revision; mHealth, mobile health. 
detrimental impact on language outcomes, school performance and vocational opportunities. ${ }^{1-9}$ We hypothesise that the mHealth screening protocol will be more sensitive than the current school screening protocol, more accurately identifying children with hearing loss because it includes a middle ear assessment that is essential to fully evaluating ear health in a population with high prevalence of otitis media (hypothesis 2).

Prevalence of hearing loss as defined by the WHO (PTA $>25 \mathrm{~dB}$ at $0.5 \mathrm{kHz}, 1 \mathrm{kHz}, 2 \mathrm{kHz}$ and $4 \mathrm{kHz}$ in either ear) will be compared from the first and second annual audiometric assessments. ${ }^{48}$ We hypothesise that hearing loss prevalence will decrease in telemedicine referral communities compared with primary care referral communities due to expedited diagnosis and treatment (hypothesis 3a).

Hearing-related quality of life will be measured using HEAR-QL child self-report. ${ }^{49}{ }^{50}$ HEAR-QL has been selected, because it is the only validated tool available for hearing-specific quality of life in children 7-18years of age. This study represents the first use of HEAR-QL in Alaska Natives and an opportunity to extend utilisation of this instrument to the Alaska Native population. We have obtained feedback from our initial focus groups on consequences of hearing loss that are specific to Alaskan Native culture. Based on this feedback, we have developed a short supplemental section to the HEAR-QL questionnaire to capture culture-specific quality of life (eg, willingness to participate in activities such as hunting or use of motor vehicles). We hypothesise that hearing-related quality of life measures will improve from year 1 to year 2 in telemedicine referral communities compared with primary care referral (hypothesis $3 \mathrm{~b}$ ).

School performance will be assessed using AIMSweb, a validated, reliable measure of math and reading performance that is administered to all children in kindergarten to 8th grade three times annually in BSSD.${ }^{51}{ }^{52}$ We hypothesise that school performance will improve from year 1 to year 2 in telemedicine referral communities compared with primary care referral communities due to expedited diagnosis and treatment (hypothesis 3c).

\section{Timing of data collection}

The timing of data collection is detailed in figure 3 . The qualitative components of the study illustrated in figure 3 are discussed in the companion protocol. ${ }^{34}$ The study team will administer the mHealth screening, audiometric assessment and HEAR-QL in every community once annually for two consecutive academic years. AIMSweb is administered by schools three times annually. These data will be released to the study team as it becomes available.

\section{Sample size}

There are approximately 1900 students enrolled in the BSSD across 15 communities (BSSD, personal communication, 2016). We anticipate that total accrual in the study of $80 \%$ of children who attend BSSD schools $(n=1520)$ is feasible because the school district already participates in state-mandated annual school hearing screening. Once $50 \%$ of parental consents have been obtained in all communities, the randomisation process will occur so the research team can plan for logistics for the referrals. This

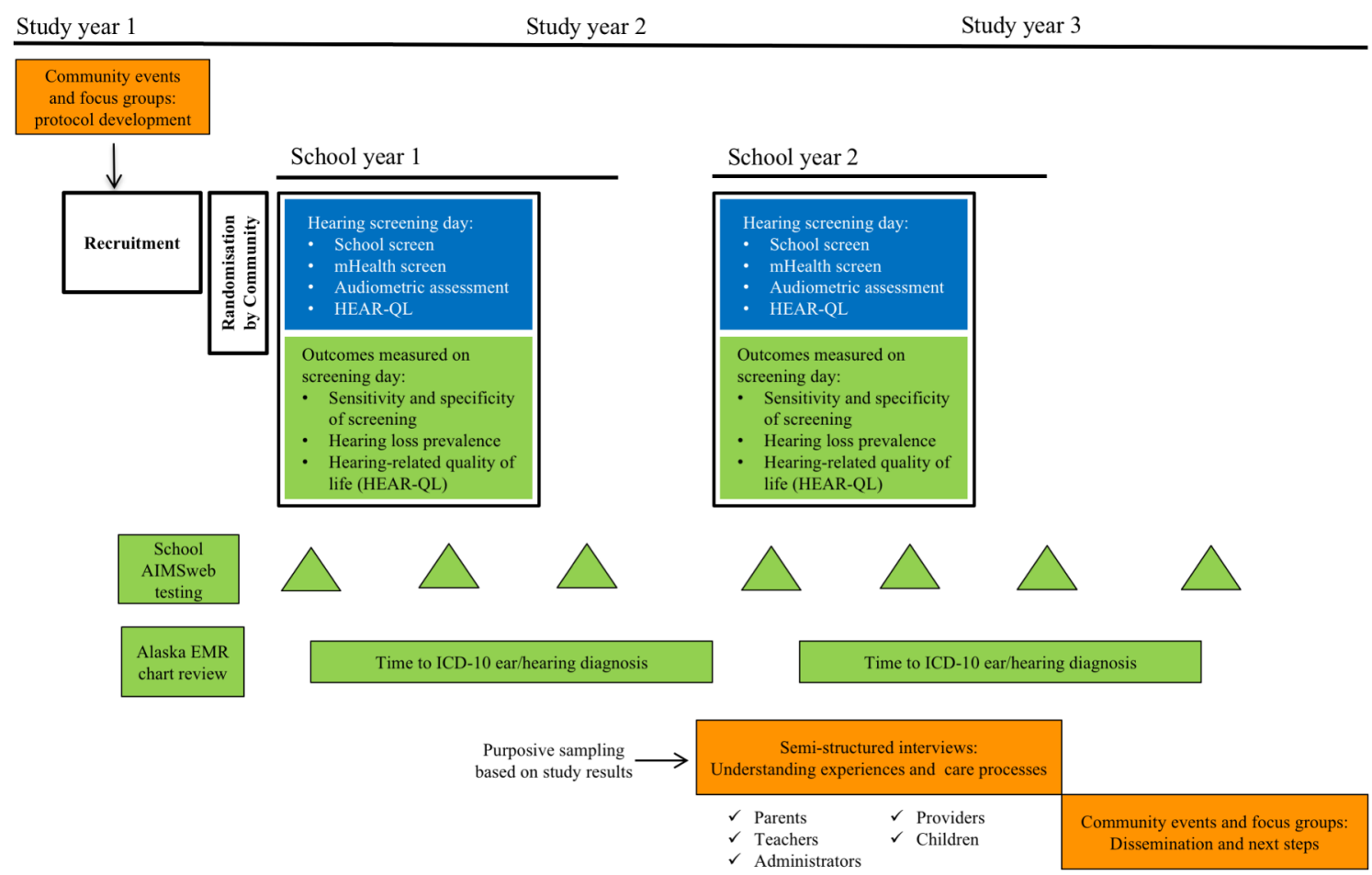

Figure 3 Timing of qualitative and quantitative data collection in the Hearing Norton Sound comparative effectiveness community randomised trial. EMR, electronic medical record; ICD-10, International Statistical Classification of Diseases, 10th Revision; mHealth, mobile health. 
study is intended to be inclusive of all children in BSSD, and thus we will continue to accept parental consents until school screening day, which represents the last day the randomised referral assignment can be strictly concealed. All children who assent to participation, have parental consent and are present on the day of screening will be enrolled. Maximum enrolment is therefore expected to be 1900 children.

Power calculations were performed for an assumed fixed sample size $(n=1500)$. For the primary outcome of time to diagnosis, our preliminary studies have indicated that wait times decreased from an average of 4.2 months (18weeks) to 2.5 months (10 weeks) when telemedicine was implemented in village health clinics in the Norton Sound region, with a SD of 0.7 months ${ }^{16}$ Based on these data, we hypothesise that expedited telemedicine referral will reduce wait times by 8 weeks compared with current primary care referral. Assuming $80 \%$ power, type 1 error of 0.05 , intracluster correlation coefficient (ICC) of 0.25 and $10 \%$ loss to follow-up, 15 clusters will provide $81 \%$ power to assess a difference in absolute mean reduction in time to diagnosis of 3 weeks between arms. If we assume ICC $=0.05$, then we will have $80 \%$ power to detect a difference in mean reduction in time to diagnosis of 1.5 weeks between arms. In general, we will have sufficient power to detect a mean difference in effect size of 0.8 and 0.4 between arms for any continuous secondary outcomes assuming ICC $=0.25$ and 0.05 , respectively. Assuming $20 \%$ prevalence of hearing loss (300 children with confirmed hearing loss by audiometric assessment) and type I error $=0.05$, we will have $80 \%$ power to detect a sensitivity of $96.6 \%$ in the mHealth screening versus $90 \%$ in the current screening protocol, with assumed ICC $=0.05$.

\section{Data management and security}

Data will be collected and managed using a Research Electronic Data Capture (REDCap) database hosted by Duke University School of Medicine and available only to authorised investigators at Norton Sound, Duke and Johns Hopkins. REDCap is a secure, encrypted web-based platform suitable for multi-institutional research studies that has the capability for data entry, import of data from multiple external sources, audit of data manipulation and automated export to statistical packages for analysis. Password protection will be required, and passwords will conform to Norton Sound Health Corporation (NSHC) and Duke University policies.

Linkage of patient data will be required for the randomised trial and has been approved by the institutional review boards of Alaska Area, Norton Sound and Duke University. Data sources include audiometric screening and evaluation with validated instruments, HEAR-QL questionnaires, school performance data (AIMSweb), parent sociodemographic questionnaire and chart review of the Norton Sound EMR. We will use a unique identifier (UIN) on all forms and communications to allow for data linkage across sources. Access to the key linking UIN to participant names will be restricted to the principal investigators (PIs) and authorised NSHC study personnel. The key will be password protected and maintained on a secure NSHC server.

Every effort will be made to facilitate direct entry of data into the database whenever possible. Data from hearing screening, diagnostic audiometry, tympanometry and otoscopy will be imported directly from the respective mHealth devices. Quality of life data from the HEAR-QL survey will be entered directly using an electronic survey administered via tablet. In case of technical difficulties with electronic survey administration, such as loss of internet connection at a community school, paper forms will be available as backup. Parents will be given the option to complete the sociodemographic survey by phone. For those who prefer paper, a hard copy will be included in the school intake packet. Data that cannot be directly entered electronically, including AIMSweb results and medical record abstractions on each child who receives a referral from a positive hearing screen, will be manually entered into the database. All manual data entry will be performed by study team members blinded to allocation using double data entry. Database design will incorporate all necessary logic and range checks and prompts. Double data entry will be compared, with discrepancies identified and resolved.

Paper copies of consent forms, assent forms and any paper-based HEAR-QL and sociodemographic surveys will be stored in a locked filing cabinet at Norton Sound Health Corporation, accessible only by authorised study staff.

\section{Censoring and missing data}

As we plan to screen all children in all grades annually with the current school screening protocol and mHealth screen, in year 2 , the 12 th graders from year 1 will no longer be enrolled in school, and a new kindergarten class will have matriculated. This natural progression in each academic year is representative of the school-aged population at large. Due to this design consideration, these children ( $15 \%$ of the study population) will contribute only up to 1 annual data point, with the other annual data point designated as a protocol-defined censoring event that will be considered missing at random so will not induce bias with proper analysis. Children in other gradeyears ( $\sim 85 \%$ of the study population) will contribute up to 2 annual data points.

For the primary outcome of time to diagnosis, we will capture and review all encounters documented in the multiorganisational EMR used by NSHC to determine whether a participating child sought care after screening positive (a binary outcome), and among those who sought care, the time to first ear/hearing ICD-10 diagnosis. We believe we can achieve complete data collection without any missing data for these analyses and will use mixed effects modelling to address the within-child outcome correlation for children who contributed 2 annual data points in the same part of the analyses. 
For sensitivity, specificity and prevalence estimates, the data on which we base the analyses will be available for all children with parental consent who were present at school on hearing screening day. We will carefully document and compare characteristics of school absence and dropout of those who consented and participated to those who were absent for the screening and will conduct sensitivity analyses on the prevalence estimates using multiple imputation and propensity score weighting to evaluate the robustness of results. We will also monitor absenteeism in real time during each of the four enrolment periods (fall 2017, spring 2018, fall 2018 and spring 2019). If at the end of an enrolment period missing data due to absenteeism exceeds $10 \%$, a make-up screening will be performed in communities where it is needed.

While school hearing screening is mandated by the state of Alaska, there is currently no policy in place regarding missed screening due to absenteeism from school. In some schools, rescreening is done, and in others, it is not. We plan to rescreen communities with $>10 \%$ absenteeism, with the goal of creating a rescreening policy that could be incorporated into the Alaska school screening mandate if the intervention is successful and ultimately becomes standard of care in the state.

\section{Statistical methods}

Statistical significance and software

Two-sided $\mathrm{p}$ values $<0.05$ will be considered statistically significant. All quantitative analyses will be performed with Stata V.13.1 and SAS V.9.4.

\section{Intention to treat}

All participating communities have telemedicine capabilities within village health clinics that will remain available to CHAs to use at their discretion throughout the course of the study, maintaining a real-world environment where some crossover between the study arms may occur. Data will nevertheless be evaluated using an intention-to-treat approach, with all data from intervention and control communities assessed by allocation regardless of adherence to the intervention arm. We will monitor the use of telemedicine through EMR chart reviews that will be conducted on all children screening positive.

\section{Overview of analysis}

Individual child and community characteristics will be compared to identify potential imbalances of key variables between randomised arms, and any imbalances in characteristics noted during the initial analysis will be used as adjustment variables in subsequent main analyses and other multivariable modelling. Sociodemographic information collected by parental self-report during the school intake process will facilitate any necessary confounding adjustment. Reliability of self-reported data will be confirmed in a random subset of questionnaires by comparison with socioeconomic data kept on file at Norton Sound Health Corporation. This test for reliability was selected because other individual-level surrogate measures of SES, such as qualification for free or reduced-price lunch or Medicaid eligibility, are nearly universal in participating communities and are therefore not useful for differentiating SES in the study population.

\section{Primary analyses}

The primary outcome, time to ICD-10 ear/hearing diagnosis from the date of screening, will be calculated in days from screening day. If there exist children who were lost to follow-up after screening positive, data analyses will proceed with a two-part approach: first, the per cent not receiving care will be compared between the two referral arms; next, conditioning on those who sought care, the mean time to diagnosis will be compared between the two referral groups. Outcome correlation for children who contributed 2 annual data points will be addressed using random-effects frailty models for correlated timeto-event outcomes, mixed effects modelling for continuous outcomes, and generalised estimating equations (GEEs) for binary outcomes to assess the marginal effects of the intervention. Annual time trend in mean outcome between arms will be captured by modelling the main effects of intervention and time and the interaction effect of intervention by time. Children contributing 1 or both annual data points will be included in this unified modelling approach for data analyses.

\section{Secondary analyses}

Sensitivity and specificity of the current and mHealth screening protocols will be calculated by comparing to the benchmark audiometric assessment.

Hearing loss prevalence will be calculated by community separately for year 1 and year 2 of screening. Effect of the intervention on changes in hearing loss prevalence between the two time-points will be assessed using GEE models incorporating a time by intervention interaction term, other appropriate covariates and a correlation structure reflecting both community clustering and temporal correlations using all available data. Robust estimation will be used for inferences on the marginal effects.

HEAR-QL will be compared between children with hearing loss and their normal-hearing peers. Effect of the intervention on trajectory of HEAR-QL scores over time between arms will be assessed using mixed effects models that properly account for temporal correlation and clustering by communities. To the extent possible, we will correlate HEAR-QL outcome with ear and hearing diagnosis, AIMSweb math and reading scores and other relevant outcomes to evaluate validity of this tool for Alaska Native populations.

AIMSweb math and reading scores will be compared between children with hearing loss and their normalhearing peers. Effect of the intervention on trajectory of AIMSweb scores over time between arms will be assessed using mixed effects models that properly account for temporal correlation and clustering by communities. 


\section{Heterogeneity of treatment effects}

In this rural Alaska population that experiences such high prevalence of middle ear disease, it is possible that previous hearing loss diagnosis and treatment may influence efficacy of the intervention. We have defined an ear/hearing condition under active management as an audiology or ENT encounter within 3 months of the date of screening or a child wearing a hearing aid on screening day. Previously managed conditions are defined as an audiology or ENT encounter $>3$ months prior to screening day. We will evaluate for possible heterogeneity of treatment effect (HTE) by analysing outcomes separately for children with new/previously undiagnosed conditions, actively managed conditions and previously managed conditions. The qualitative interviews described in the companion protocol will be essential for fully understanding the impact of prior diagnoses on families' response to follow-up for hearing screening referrals.

Aside from previous hearing loss diagnosis, the other main subgroup analysis for exploring HTE will be native versus non-native subgroups. We will also explore for potential HTEs by SES variables such as age, gender, caretaker education and environment. We have no other planned subgroup analyses, as the intervention is intended to be applied at the community level and not to individual subpopulations.

If any potential HTE was found, a formal statistical comparison testing treatment group by subgroup interaction through statistical modelling will be carried out to confirm statistical significance of such HTE. Subgroup treatment effects and corresponding 95\% CI will be estimated. We will also carefully examine subgroup characteristics for differences that may be related to such HTE as part of a hypothesis-generating exercise. We will interpret any potential HTE finding cautiously, including reporting the number of post hoc analyses conducted to arrive at such a finding.

\section{Data and safety monitoring}

An independent Data Safety Monitoring Board (DSMB) will review interim data from year 1 in month 20 of the 36-month study to allow for a minimum of 5-months follow-up for the children enrolled in the spring of year 1 of screening. The DSMB will be independent of the sponsor and have no competing interests in the study. The board will report to the PIs. Composition of the board will include a statistician and experts in clinical trials, hearing loss and Alaska child health. Because this is a minimal risk study and year 1 data will not be available for interim analysis until after year 2 data collection has begun, no stopping rules will be formulated.

The PIs will be responsible for ensuring participant safety and management of all data. As a minimal risk study, no serious adverse events are anticipated. Adverse events, such as unexpected physical harm to a child during the screening process (eg, a rare case of tympanic membrane perforation from a probe) or breach of data security policies will be reported directly to the PIs, who will be responsible for reporting to the IRBs of Alaska Area, Norton Sound and Duke, as appropriate. The study leadership team will meet weekly via teleconference to review study progress, data quality and participants' safety.

\section{ETHICS AND DISSEMINATION \\ Research ethics approval}

The trial protocol has been reviewed and approved by the following Institutional Review Boards: Alaska Area, Norton Sound, and Duke University. Any protocol amendments will be approved by participating IRBs and documented on ClinicalTrials.gov.

\section{Access to data}

In the spirit of supporting implementation across the state, all information on the content of the mHealth screening protocol and telemedicine referral process will be made publicly available to other regional healthcare systems in Alaska and organisations interested in implementing study findings in their region. A HIPPA compliant, de-identified dataset may be made available on reasonable written request, with necessary approvals and possible execution of a formal data use agreement, if appropriate.

\section{Dissemination plan}

Study results will be distributed in multiple ways that highlight equal emphasis on scientific and community dissemination. The trial has been registered on clinicaltrials.gov (NCT03309553), and results will be submitted to the database within 1 year of completion. Results will be published in peer-reviewed journals and presented at academic conferences. We will follow the Consolidated Standards of Reporting Trials 2010 guidelines for reporting parallel group randomised trials in publications related to this study. Sharing results and updates about the project in real time with Alaska communities is similarly a priority for our study team and will be spearheaded by our communications outreach specialist. Results will be disseminated in tribal-related conferences and through other sources that are specific to the cultural environment of Alaska.

\section{Limitations}

There are several study limitations that should be mentioned. Due to the nature of the intervention as a referral process, it is impossible to mask children and parents to allocation after the school screening day, but masking will be maintained through the screening. Study team members performing outcome assessments will be masked to referral allocation. Team members performing data collection will be masked to the results of each hearing screen and audiometric evaluation.

Screening and referral interventions are by nature meant to be applied to the community as a whole, so the cluster randomised design is a logical choice. However, a 
cluster randomised trial has lower statistical power and precision than an individually randomised trial with the same number of individual participants and has to address typical challenges such as selection bias, imbalance between study arms and generalisability accompanying a cluster randomised design. Based on preliminary data, we expect a sizeable intervention effect on the primary outcome, which we will have ample statistical power to detect with our proposed sample size. We plan to rigorously collect key variables associated with study outcomes so we can address potential imbalances between study arms due to cluster randomisation or potential selection bias due to selected participation. We can use a statistical approach to evaluate generalisability of intervention effects based on characteristics of our trial sample and the characteristics of the target population to which the results are to be generalised.

We have hypothesised that prevalence of hearing loss will decrease in communities randomised to telemedicine referral due to expedited treatment. This is based on the assumption that infection-mediated aetiologies of hearing loss, such as chronic otitis media, are the predominant factors influencing hearing loss prevalence in participating communities. It is possible, however, that prevalence of hearing loss will actually increase in participating communities due to enhanced diagnosis from the mHealth screening and audiometric assessments conducted on screening day across the study population. It is therefore important to have rigorous data collection during the second school year to evaluate the potential impact on hearing loss compared with year 1 data.

Our hearing-related quality of life and school performance outcomes are only available in select age groups, with HEAR-QL validated in age 7 years and older and AIMSweb administered in kindergarten to eighth grade. We are nevertheless including all age groups in the study, as the intention is to maintain a real-world environment where all children are eligibile to participate.

There is potential for substantial crossover between study arms for several reasons. This region of Alaska was selected for the study in part because of its consistent use of telemedicine. This also means that telemedicine will be available to CHAs in both study arms and can be used at their discretion even in control communities, just as standard practice has been in these communities prior to study initiation. Because we have spent approximately 6 months hosting community events and focus groups and raising awareness about childhood hearing loss through media outreach in participating communities, we may influence behaviour of parents, children, educators and CHAs. We will monitor the use of telemedicine in all communities to better understand how behaviour has changed as a result of the study. Regardless of crossover, data will be analysed with an intention-to-treat approach.

\section{Potential contributions of this study}

Early identification and treatment is essential for minimising impact of childhood hearing loss, which has known effects on language development, school achievement, future employment opportunities and quality of life. ${ }^{1-9} 4950$ Early identification and treatment are particularly challenging in remote environments, where hearing loss prevalence is high and access to ear and hearing care is traditionally limited.

This study will provide the first empirical evidence on the effectiveness of current school screening programmes in the state of Alaska and represents a unique opportunity to evaluate a new school screening protocol that uses mHealth-based screening tools and telemedicine referral in a remote region of Alaska where telemedicine has already been standard practice for triage of health concerns for over 15 years. A strength of this study is that the intervention was developed with Alaska stakeholder and community input, including parents, teachers, administrators, CHAs, children and community leaders, to ensure that it represents a culturally relevant solution to address undiagnosed childhood hearing loss in Alaska Native communities. The intervention purposefully builds on processes already in place in the Alaska school and healthcare systems to increase scalability within the state. Based on results of the trial, an evidence-based screening protocol could be implemented in a standardised fashion statewide, addressing childhood hearing loss across Alaska.

In addition to its role in Alaska, the concept of mHealth-based hearing screening with telemedicine referral is broadly applicable outside the state to address barriers to early identification and treatment in remote environments. While aspects of this screening intervention are designed specifically for the Alaskan context, the combination of mHealth screening and telemedicine referral could be replicated to address childhood hearing loss disparities in other remote regions of the world.

\section{Author affiliations}

${ }^{1}$ Department of Surgery, Duke University School of Medicine, Durham, North Carolina, USA

${ }^{2}$ Duke Global Health Institute, Durham, North Carolina, USA

${ }^{3}$ Department of Audiology, Norton Sound Health Corporation, Nome, Alaska, USA

${ }^{4}$ Department of Medicine, Johns Hopkins University School of Medicine, Baltimore, Maryland, USA

${ }^{5}$ Departments of Biostatistics and Epidemiology, Johns Hopkins Bloomberg School of Public Health, Baltimore, Maryland, USA

${ }^{6}$ Departments of International Health and Epidemiology, Johns Hopkins University Bloomberg School of Public Health, Baltimore, Maryland, USA

${ }^{7}$ Department of Mental Health, Johns Hopkins Bloomberg School of Public Health, Baltimore, Maryland, USA

${ }^{8}$ Norton Sound Health Corporation, Nome, Alaska, USA

Acknowledgements We would like to acknowledge the Alaska stakeholder team for their contributions to the project: Laureli Ivanoff (Communications Outreach Specialist), Paul 'Bebucks' Ivanoff (Lead Parent Stakeholder), John Kokesh (Lead ENT Stakeholder), Stephanie McConnell (Lead Patient Stakeholder) and Patricia 'Patty' Vink (Lead Education Stakeholder). Most of all, we thank the participants, whose support is vital to the success of this community-based endeavor.

Contributors PH and SDE are the principal investigators and direct all study activities and coordinate team members. Study design: all authors. Analysis design: all authors. Protocol generation: all authors. 
Funding This project is supported by the Patient Centered Outcomes Research Institute (AD-1602-34571). The sponsor of this study is Norton Sound Health Corporation (PO Box 966 Nome, AK 99762; (907) 443-3311).

Disclaimer Neither the study sponsor nor the funder have a role in study design, collection, management, analysis and interpretation of data; writing of the report; or the decision to submit for publication.

Competing interests None declared.

Patient consent Obtained.

Ethics approval The trial protocol has been reviewed and approved by the following Institutional Review Boards: Alaska Area, Norton Sound and Duke University.

Provenance and peer review Not commissioned; externally peer reviewed.

Open access This is an open access article distributed in accordance with the Creative Commons Attribution Non Commercial (CC BY-NC 4.0) license, which permits others to distribute, remix, adapt, build upon this work non-commercially, and license their derivative works on different terms, provided the original work is properly cited, appropriate credit is given, any changes made indicated, and the use is non-commercial. See: http://creativecommons.org/licenses/by-nc/4.0/.

\section{REFERENCES}

1. Wake M, Hughes EK, Poulakis Z, et al. Outcomes of children with mild-profound congenital hearing loss at 7 to 8 years: a population study. Ear Hear 2004;25:1-8.

2. Kennedy CR, McCann DC, Campbell MJ, et al. Language ability after early detection of permanent childhood hearing impairment. $N$ Engl J Med 2006;354:2131-41.

3. Tomblin JB, Harrison M, Ambrose SE, et al. Language outcomes in young children with mild to severe hearing loss. Ear Hear 2015;36 Suppl 1:76S-91.

4. Järvelin MR, Mäki-Torkko E, Sorri MJ, et al. Effect of hearing impairment on educational outcomes and employment up to the age of 25 years in northern Finland. Br J Audiol 1997;31:165-75.

5. Bess FH, Dodd-Murphy J, Parker RA. Children with minimal sensorineural hearing loss: prevalence, educational performance, and functional status. Ear Hear 1998;19:339-54.

6. Lieu JE, Tye-Murray N, Fu Q. Longitudinal study of children with unilateral hearing loss. Laryngoscope 2012;122:2088-95.

7. Khairi Md Daud M, Noor RM, Rahman NA, et al. The effect of mild hearing loss on academic performance in primary school children. Int J Pediatr Otorhinolaryngol 2010;74:67-70.

8. Emmett SD, Francis HW. The socioeconomic impact of hearing loss in U.S. adults. Otol Neurotol 2015;36:545-50.

9. Jung $D$, Bhattacharyya N. Association of hearing loss with decreased employment and income among adults in the United States. Ann Otol Rhinol Laryngol 2012;121:771-5.

10. Reed D, Struve S, Maynard JE. Otitis media and hearing deficiency among Eskimo children: a cohort study. Am J Public Health Nations Health 1967;57:1657-62.

11. Singleton RJ, Holman RC, Plant R, et al. Trends in otitis media and myringtomy with tube placement among American Indian/Alaska native children and the US general population of children. Pediatr Infect Dis J 2009;28:102-7.

12. Smith DF, Boss EF. Racial/ethnic and socioeconomic disparities in the prevalence and treatment of otitis media in children in the United States. Laryngoscope 2010;120:2306-12.

13. Niskar AS, Kieszak SM, Holmes A, et al. Prevalence of hearing loss among children 6 to 19 years of age: the Third National Health and Nutrition Examination Survey. JAMA 1998;279:1071-5.

14. Curns AT, Holman RC, Shay DK, et al. Outpatient and hospital visits associated with otitis media among American Indian and Alaska native children younger than 5 years. Pediatrics 2002;109:e41-E41.

15. Carroll M, Cullen T, Ferguson S, et al. Innovation in Indian healthcare: using health information technology to achieve health equity for American Indian and Alaska Native populations. Perspect Health Inf Manag 2011;8:1d.

16. Hofstetter PJ, Kokesh J, Ferguson AS, et al. The impact of telehealth on wait time for ENT specialty care. Telemed J E Health 2010;16:551-6.

17. Golnick C, Asay E, Provost E, et al. Innovative primary care delivery in rural Alaska: a review of patient encounters seen by community health aides. Int J Circumpolar Health 2012;71:18543.

18. Overview of the Alaska Community Health Aide Program. 2005:1-8. Available at http://www.akchap.org/resources/chap_library/Referral Physician/CHAM_CHAP_Overview.pdf (Accessed 2 Mar 2016).
19. Kokesh J, Ferguson AS, Patricoski C. The Alaska experience using store-and-forward telemedicine for ENT care in Alaska. Otolaryngol Clin North Am 2011;44:1359-74.

20. Hays H, Carroll M, Ferguson S, et al. The Success of Telehealth Care in the Indian Health Service. Virtual Mentor 2014;16:986-96.

21. Kokesh J, Ferguson AS, Patricoski C, et al. Traveling an audiologist to provide otolaryngology care using store-and-forward telemedicine. Telemed J E Health 2009;15:758-63.

22. Kokesh J, Ferguson AS, Patricoski C. Telehealth in Alaska: delivery of health care services from a specialist's perspective. Int J Circumpolar Health 2004;63:387-400.

23. Kokesh J, Ferguson AS, Patricoski C. Preoperative planning for ear surgery using store-and-forward telemedicine. Otolaryngol Head Neck Surg 2010;143:253-7.

24. Kokesh J, Ferguson AS, Patricoski C, et al. Digital images for postsurgical follow-up of tympanostomy tubes in remote Alaska. Otolaryngol Head Neck Surg 2008;139:87-93.

25. Patricoski C, Kokesh J, Ferguson AS, et al. A comparison of inperson examination and video otoscope imaging for tympanostomy tube follow-up. Telemed J E Health 2003;9:331-44.

26. Alaska Statutes. 2015. Available at http://www.legis.state.ak.us/ basis/statutes.asp\#14.30.127 (Accessed 8 Jan 2016).

27. Kaplan GJ, Fleshman JK, Bender TR, et al. Long-term effects of otitis media: a ten-year cohort study of Alaskan Eskimo children. Pediatrics 1973:52:577-85.

28. Bowd AD. Otitis media: health and social consequences for aboriginal youth in Canada's north. Int J Circumpolar Health 2005;64:5-15.

29. Mehra S, Eavey RD, Keamy DG. The epidemiology of hearing impairment in the United States: newborns, children, and adolescents. Otolaryngol Head Neck Surg 2009;140:461-72.

30. Singleton R, Seeman S, Grinnell M, et al. Trends in otitis media and myringotomy with tube placement among american indian and alaska native children and the us general population of children after introduction of the 13-valent pneumococcal conjugate vaccine. Pediatr Infect Dis J 2018;37:e6-12.

31. Owen MJ, Baldwin CD, Swank PR, et al. Relation of infant feeding practices, cigarette smoke exposure, and group child care to the onset and duration of otitis media with effusion in the first two years of life. J Pediatr 1993;123:702-11.

32. Hennessy TW, Bressler JM. Improving health in the Arctic region through safe and affordable access to household running water and sewer services: an Arctic Council initiative. Int J Circumpolar Health 2016;75:31149.

33. Subcommittee on Childhood Hearing Screening. American Academy of Audiology childhood hearing screening guidelines. 2011. Available at http://www.cdc.gov/ncbddd/hearingloss/documents/ aaa_childhood-hearing-guidelines_2011.pdf (Accessed 10 Jan 2016).

34. Emmett SD, Robler SK, Gallo JJ, et al. Hearing Norton Sound: Mixed Methods Protocol of a Community Randomized Trial to Address Childhood Hearing Loss in Rural Alaska. BMJ Open 2018.

35. Gorga MP, Neely ST, Ohlrich B, et al. From laboratory to clinic: a large scale study of distortion product otoacoustic emissions in ears with normal hearing and ears with hearing loss. Ear Hear 1997;18:440-55.

36. Prieve BA, Schooling T, Venediktov R, et al. An evidence-based systematic review on the diagnostic accuracy of hearing screening instruments for preschool- and school-age children. Am J Audiol 2015;24:250-67.

37. New Zealand Health Techology Assessment. Screening programmes for the detection of otitis media with effusion and conductive hearing loss in pre-school and new entrant school children: a critical approasial of the IIterature. Christchurch NZ: New Zealand Health Technology Assessment Clearing House, 1998.

38. FitzZaland RE, Zink GD. A comparative study of hearing screening procedures. Ear Hear 1984;5:205-10.

39. Mahomed-Asmail F, Swanepoel deW, Eikelboom $\mathrm{RH}$, et al. Clinical Validity of hearScreen ${ }^{\mathrm{TM}}$ Smartphone Hearing Screening for Schoo Children. Ear Hear 2016;37:e11-e17.

40. Yousuf Hussein S, Wet Swanepoel D, Biagio de Jager L, et al. Smartphone hearing screening in mHealth assisted communitybased primary care. J Telemed Telecare 2016;22:405-12.

41. Swanepoel deW, Myburgh HC, Howe DM, et al. Smartphone hearing screening with integrated quality control and data management. Int J Audiol 2014;53:841-9.

42. Yousuf Hussein S, Swanepoel W, Mahomed F, et al. Communitybased hearing screening for young children using an mHealth service-delivery model. Glob Health Action 2018;11:1467077.

43. Thompson GP, Sladen DP, Borst BJ, et al. Accuracy of a tablet audiometer for measuring behavioral hearing thresholds in a clinical population. Otolaryngol Head Neck Surg 2015;153:838-42. 
44. American National Standards Institute. Methods for manual puretone threshold audiometry. New York: American National Standards Institute, 1978

45. American Speech-Language Hearing Association. Guidelines for manual pure-tone threshold audiometry. Rockville, MD, 2005.

46. American Speech-Language Hearing Association. Tympanometry. 1988. Available at https://www.asha.org/policy/rp1988-00027/ (Accessed 13 Mar 2018).

47. British Society of Audiology. Recommended Procedure for Tympanometry. 2013. Available at http://www.thebsa.org.uk/wpcontent/uploads/2014/04/BSA_RP_Tymp_Final_21Aug13_Final.pdf/ (Accessed 13 Mar 2018).
48. World Health Organization. Grades of hearing impairment. 2016. Available at http://www.who.int/pbd/deafness/hearing_impairment_ grades/en/ (Accessed 28 Feb 2016).

49. Umansky AM, Jeffe DB, Lieu JE. The HEAR-QL: quality of life questionnaire for children with hearing loss. J Am Acad Audiol 2011;22:644-53.

50. Rachakonda T, Jeffe DB, Shin JJ, et al. Validity, discriminative ability, and reliability of the hearing-related quality of life questionnaire for adolescents. Laryngoscope 2014;124:570-8.

51. Pearson Education.. AIMSweb ${ }^{\circledR}$ Test of Early Literacy Administration and Scoring Guide. Bloomington, MN: NCS Pearson, 2012.

52. Pearson Education. AIMSweb Technical Manual. Bloomington MN: NCS Pearson, 2012. 